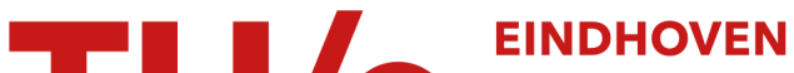 \\ UNIVERSITY OF \\ TECHNOLOGY
}

\section{Similarity-based adaptive complementary filter for IMU fusion}

Citation for published version (APA):

Andriën, A. R. P., Guerreiro Tomé Antunes, D. J., van de Molengraft, M. J. G., \& Heemels, W. P. M. H. (2018). Similarity-based adaptive complementary filter for IMU fusion. 3044-3049. Paper presented at 16th European

Control Conference, ECC 2018, Limassol, Cyprus. https://doi.org/10.23919/ECC.2018.8550358

DOI:

10.23919/ECC.2018.8550358

Document status and date:

Published: 27/11/2018

Document Version:

Author's version before peer-review

Please check the document version of this publication:

- A submitted manuscript is the version of the article upon submission and before peer-review. There can be important differences between the submitted version and the official published version of record. People interested in the research are advised to contact the author for the final version of the publication, or visit the $\mathrm{DOI}$ to the publisher's website.

- The final author version and the galley proof are versions of the publication after peer review.

- The final published version features the final layout of the paper including the volume, issue and page numbers.

Link to publication

\section{General rights}

Copyright and moral rights for the publications made accessible in the public portal are retained by the authors and/or other copyright owners and it is a condition of accessing publications that users recognise and abide by the legal requirements associated with these rights.

- Users may download and print one copy of any publication from the public portal for the purpose of private study or research.

- You may not further distribute the material or use it for any profit-making activity or commercial gain

- You may freely distribute the URL identifying the publication in the public portal.

If the publication is distributed under the terms of Article $25 \mathrm{fa}$ of the Dutch Copyright Act, indicated by the "Taverne" license above, please follow below link for the End User Agreement:

www.tue.nl/taverne

Take down policy

If you believe that this document breaches copyright please contact us at:

openaccess@tue.nl

providing details and we will investigate your claim. 


\title{
Similarity-Based Adaptive Complementary Filter for IMU Fusion
}

\author{
A.R.P. Andriën, D. Antunes, M.J.G. van de Molengraft, W.P.M.H. Heemels
}

\begin{abstract}
This paper addresses the attitude estimation problem using vector and gyroscope measurements. We propose a novel adaptation scheme for the complementary filter cut-off frequency that is based on the similarity between independent estimates obtained from the vector and gyroscope measurements. The adaptive complementary filter is also derived on the special orthogonal group and convergence of the filter is established. The effectiveness of our approach is demonstrated with simulation results.
\end{abstract}

\section{INTRODUCTION}

Estimating the attitude of a rigid body based on sensor data from an Inertial Measurement Unit (IMU), consisting of (three-axes) accelerometers, gyroscopes and magnetometers, is crucial in many applications and in particular in the context of Unmanned Aerial Vehicles (UAVs). As a result much work has been developed on attitude estimation over the years [1]-[13]. However, the attitude estimation problem has gained a renewed interest recently. In fact, the trend in the last few years in the UAV industry is that of size and cost reduction. Therefore, it is often the case that the available data from low-cost IMUs does not provide sufficiently accurate information for the current estimation techniques to deliver the desired attitude accuracy [13].

The most popular IMU-based attitude estimation approaches are the Kalman filter (KF) and the complementary filter (CF) [1]. There are many variants of the Kalman filter, such as linear [2], [3], extended Kalman filters (EKFs) [4][7] and unscented Kalman filters (UKFs) [8], [9]. Some of the advantages of linear KFs are their effectiveness and low implementation cost, as well as the guaranteed optimality and stability under the assumption of linear process and measurement models as well as Gaussian noise and disturbance processes. However, for nonlinear processes the EKF and UKF typically show better results, at the cost of more computational complexity and the absence of stability guarantees. In the context of complementary filters, one of the first applications of the linear CF to attitude estimation was presented in [10]. Nonlinear CFs that operate directly on the Special Orthogonal Group ( $\mathrm{SO}(3)$ ) have also been developed and successfully implemented in [11], [12] and [13]. One of the main advantages of the CFs over KFs is the fact that they do not require an explicit model of the dynamics and/or sensors, which is hard to obtain in many applications and in particular in the context of small-scale UAVs, where some aerodynamic effects such as turbulence, drag and ground effects have a significant effect but are hard to model. Another advantage is its simplicity, since it relies on simple low and high pass filtering of the sensor data.

The idea behind the complementary filter is to combine attitude estimates obtained using only vector measurements derived from the accelerometer and magnetometer, relying on on-board measurements of the gravitational acceleration and magnetic field vectors, and attitude estimates using only the gyroscope. Integrating the gyroscope provides attitude estimates with accurate high frequency content, but poor low frequency content due to integration of low-frequency noise resulting in a time-varying bias. In turn, the vector measurements are based on the assumption that the accelerometer and magnetometer only measure the gravitational vector and earth magnetic field, respectively. Under this assumption the noise of the vector measurements contains mainly high frequency content resulting from vibrations and sensor limitations. The complementary filter combines high-pass filtered estimates from the gyroscope and low-pass filtered attitude estimates from the vector measurements. In the simplest version of the complementary filter both high-pass and low-pass filters are first order and determined by a single parameter, the cut-off frequency. The smaller (larger) the cutoff frequency, the more the attitude estimate relies on the gyroscope (vector) measurements.

The complementary filter is a simple time-invariant filter, both in the linear [10] and nonlinear versions [11], [13], which performs well under the above stated assumption that the accelerometer and magnetometer only measure the gravitational vector and earth magnetic field, respectively. However, under the effect of body accelerations and magnetic disturbances caused by the environment, this assumption is no longer valid, resulting in poor attitude estimates (see Figure 2). Therefore it is reasonable to expect that adaptive filtering [14], meant to cope with signals with time-varying frequency content, could potentially lead to better performance. While the work in this direction is limited, there are some recent contributions for UAV attitude estimation, which have focused on the adaptation of the filters in order to account for the shortcomings in the sensors. For instance, in [15] an adaptive linear KF is proposed, where the measurement noise covariance matrix is adapted based on the difference between the accelerometer measurements and the gravity vector. In [16] and [17] adaptive EKFs are proposed, where the adaptation is based on the deviation of the actual from the expected measurement and the same deviation used in a fuzzy logic setting, respectively. Adaptive complementary filters (ACFs) have also been used as in [18], [19] and [20], where adaptation based on comparing the accelerometer with the gravity vector is used. Furthermore, [21] provides a complementary filter in the least squares sense (also known as Wahba's problem [22]), using the difference between the output of the filter and the measurement by the 


\section{CONFIDENTIAL. Limited circulation. For review only.}

accelerometer for adaptation and [23] proposes a multiple model adaptive complementary filter, with adaptation based on the same measure.

In this paper we propose a new adaptation rule for changing the parameter of the complementary filter online, determining which measurement (either from the gyroscope or from the vector) is more relevant for the attitude estimate. The rationale behind our approach is that the attitude estimate should rely on vector measurements if the independent estimates using only vector measurements and only gyroscope measurements are similar over a time window, possibly apart from a constant factor due to low-frequency noise of the gyroscope estimate. Otherwise the attitude estimate should rely instead on the gyroscope, since this is an indication that significant disturbances are affecting the vector measurements. The main difference in our method compared to others in the literature is the manner in which the complementary filter gain is adapted. In our case it relies on a similarity measure; to assert similarity the signals (estimates from vector and gyroscopes) must be considered over a moving time window of immediate past data. Moreover, we show that this adaptation concept is not only applicable for estimating single angles with linear models but also for estimating rotational matrices considering the non-linear kinematic model in $\mathrm{SO}(3)$. In fact, we extend the work in [13] to this adaptation setting and show that the convergence proofs given there can be easily extended to the proposed setting in this paper as well. We illustrate the effectiveness of our approach through simulation results using a full nonlinear model of a quadcopter and IMU sensors.

The contribution of the paper is therefore twofold. First we propose a novel adaptation rule for complementary filters based on the similarity between gyroscope and vector estimates; second we establish convergence of this filter.

The remainder of the paper is organized as follows. The standard attitude estimation framework is described in Section II where we also motivate the need for an adaptive approach. In Section III we introduce the proposed method considering linear models and in Section IV our method is extended to the special orthogonal group, for which we show convergence. Section V shows the main advantages over the non-adaptive filter using simulations and Section VI contains concluding remarks and directions for future work.

\section{BACKGROUND \& MOTIVATION}

Let $\mathcal{I}$ denote an inertial world fixed frame with $z$-axis aligned with the gravity vector, but pointing upwards, and the IMU fixed frame be denoted by $\mathcal{B}$, which will be referred to as the body-fixed frame. Moreover, let $R \in S O(3)$ denote the rotation matrix from $\mathcal{B}$ to $\mathcal{I}$ and $\omega=\left[\omega_{x}, \omega_{y}, \omega_{z}\right]^{T} \in \mathbb{R}^{3}$ denote the angular velocity of $\mathcal{B}$ with respect to $\mathcal{I}$ expressed in $\mathcal{B}$. Then the attitude kinematics are given by

$$
\dot{R}=R \omega_{\times}
$$

where $a_{\times}$denotes the skew symmetric matrix of $a$ such that $a_{\times} v=a \times v$ for all vectors $a$ and $v$ and $\operatorname{vex}(\cdot)$ denotes the inverse operation, so that $\operatorname{vex}\left(a_{\times}\right)=a$. The rotation matrix $R$ can be parametrized by the three $x-y-z$ Tait-Bryan angles rotating around the axes of the body frame of the UAV, namely roll $(\phi)$, pitch $(\theta)$ and yaw $(\psi)$. The attitude kinematics in these coordinates are given by

$$
\dot{\lambda}=Q(\lambda) \omega
$$

where $\lambda=[\phi, \theta, \psi]^{T} \in \mathbb{R}^{3}$ is the angle vector and $Q(\lambda)$ is given by

$$
Q(\lambda)=\left[\begin{array}{ccc}
1 & s_{\phi} t_{\theta} & c_{\phi} t_{\theta} \\
0 & c_{\phi} & -s_{\phi} \\
0 & \frac{s_{\phi}}{c_{\theta}} & \frac{c_{\phi}}{c_{\theta}}
\end{array}\right]
$$

where $c_{\epsilon}, s_{\epsilon}$ and $t_{\epsilon}$ denote the cosine, sine and tangent of an angle $\epsilon$, respectively. Note that for small angles we have $Q(\lambda) \approx I$, and we obtain a linear model. This linear model is often considered in practice and will be used in the sequel to illustrate our ideas.

The accelerometer measures body accelerations in the three axes in $\mathcal{B}$ (neglecting Coriolis effects), as given by

$$
a_{\mathcal{B}}=R^{T} \ddot{\rho}-R^{T}\left[\begin{array}{l}
0 \\
0 \\
g
\end{array}\right]+n_{a},
$$

where $\ddot{\rho} \in \mathbb{R}^{3}$ are the body accelerations in $\mathcal{I}, g$ is the Earth's gravitational acceleration and $n_{a}$ is the accelerometer noise.

The magnetometer measures the magnetic field in the body-frame, which is characterized by

$$
m_{\mathcal{B}}=R^{T} m_{I}+d_{m}+n_{m}
$$

where $m_{I} \in \mathbb{R}^{3}$ is the Earth's magnetic field in the inertial frame, $n_{m} \in \mathbb{R}^{3}$ is the magnetometer noise and $d_{m} \in \mathbb{R}^{3}$ contains the magnetic disturbances caused by the environment, such as electric motors and nearby ferromagnetic materials.

The accelerometer and magnetometer measurements can be combined to give an algebraic estimate of the angle vector. In fact, we start by noticing that by assuming negligible accelerations and magnetic disturbances we have that

$$
a_{B} \approx-R^{T}\left[\begin{array}{l}
0 \\
0 \\
g
\end{array}\right], \quad m_{B} \approx R^{T} m_{I}
$$

The rotation matrix parametrized by the roll-pitch-yaw angles is given by:

$$
R=\left[\begin{array}{ccc}
c_{\theta} c_{\psi} & c_{\psi} s_{\theta} s_{\phi}-c_{\phi} s_{\psi} & s_{\phi} s_{\psi}+c_{\phi} c_{\psi} s_{\theta} \\
c_{\theta} s_{\psi} & c_{\phi} c_{\psi}+s_{\theta} s_{\phi} s_{\psi} & c_{\phi} s_{\theta} s_{\psi}-c_{\psi} s_{\phi} \\
-s_{\theta} & c_{\theta} s_{\phi} & c_{\theta} c_{\phi}
\end{array}\right]
$$

Then, estimates for the roll and pitch angles can be directly determined from the accelerometer measurement as

$$
\begin{aligned}
& \hat{\phi}_{v}=\operatorname{atan} 2\left(a_{B, y}, a_{B, z}\right), \\
& \hat{\theta}_{v}=-\operatorname{atan} 2\left(a_{B, x}, \sqrt{a_{B, y}^{2}+a_{B, z}^{2}}\right),
\end{aligned}
$$

where atan 2 denotes the four-quadrant arctangent. Using these estimates the measured magnetic field vector is rotated as

$$
m_{\mathcal{B}}^{*}=R_{y}\left(\hat{\theta}_{a}\right) R_{x}\left(\hat{\phi}_{a}\right) m_{\mathcal{B}}
$$


and the yaw angle is subsequently estimated as

$$
\hat{\psi}_{v}=-\operatorname{atan} 2\left(m_{\mathcal{B}, y}^{*}, m_{\mathcal{B}, x}^{*}\right) \text {. }
$$

This results in a direct estimate of the angle vector as

$$
\lambda_{v}=\lambda+n_{v}
$$

where $n_{v} \in \mathbb{R}^{3}$ is considered non-stationary noise. The non-stationary behavior results from the assumption that the accelerometer only measures the gravity vector, which is valid only at slow accelerations in near-hover, and magnetic disturbances caused by the environment and the motors of the quadcopter are negligible. This results in correlated, high frequency noise for the angle vector measurements.

An estimate of the angular velocities is given by the gyroscope as

$$
\omega_{g}=\omega+n_{\omega}+n_{\text {bias }},
$$

where $n_{\omega} \in \mathbb{R}^{3}$ is considered white noise and $n_{\text {bias }}$ is a sensor bias. By assuming small angles and integrating the angular rate measurements according to (2) another estimate for the angle vector, denoted as $\lambda_{g}$, can be obtained. However, even when assuming $n_{\text {bias }}=0$, due to the white noise component in (13) this estimate will be corrupted by a random walk signal $b(t)$ leading to

$$
\lambda_{g}=\lambda+b(t)
$$

which in practice leads to a poor low-frequency estimate of $\lambda$ using this method, often interpreted as a time-varying offset/bias. Note that we assume here that there are no other disturbances, e.g. device failure, acting on the gyroscope, so that the angle estimate resulting from it can be assumed to be accurate up to the bias.

\section{A. Complementary Filter}

As mentioned previously, the complementary filter is a common method to fuse the two measurements, $\lambda_{v}$ and $\lambda_{g}$, combining the strengths of both. In the Laplace domain it takes the form

$$
\hat{\Lambda}(s)=\underbrace{\frac{C(s)}{s+C(s)}}_{F_{1}(s)} \Lambda_{v}(s)+\underbrace{\frac{s}{s+C(s)}}_{F_{2}(s)} \frac{\Omega_{g}(s)}{s},
$$

where $F_{1}$ and $F_{2}$ are a low-pass and a high-pass filter, respectively, which satisfy

$$
F_{1}(s)+F_{2}(s)=1,
$$

for any choice of the compensator $C(s)$. Schematically this can be represented as in Figure 1. Although there have been considerable efforts made in designing $C(s)$ in the literature, the most common choice is that of a simple gain $C(s)=$ $\alpha>0$, for which the filter in (15) combined with (12) and (13) results in

$$
\begin{aligned}
\hat{\Lambda}(s)= & \frac{\alpha}{s+\alpha} \Lambda_{v}(s)+\frac{s}{s+\alpha} \frac{\Omega_{g}(s)}{s} \\
= & \frac{\alpha}{s+\alpha} \Lambda(s)+\frac{s}{s+\alpha} \frac{\Omega(s)}{s}+\frac{\alpha}{s+\alpha} N_{v}(s) \\
& +\frac{s}{s+\alpha} \frac{N_{\omega}(s)}{s} .
\end{aligned}
$$

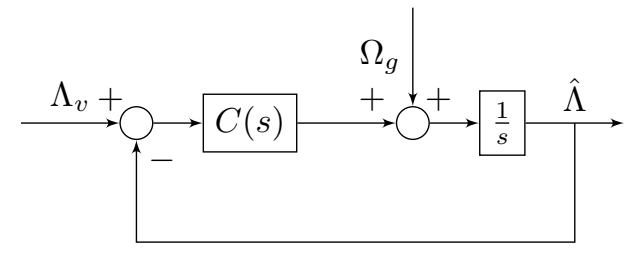

Fig. 1. Complementary filter

Note that the first two terms on the right hand side in (18) assure that the method converges in the absence of noise, whereas the two noise terms are filtered. The random walk term is high-pass filtered, which leads to low-pass filtered white noise. The term $N_{v}$, which as discussed before is assumed as mostly having high-frequency content, is lowpass filtered.

\section{B. Motivation}

If the noise $N_{v}$ would be stationary we could select a single $\alpha$ and the complementary filter would provide an adequate solution to the problem of estimating the angles. However, this noise is not stationary since:

- Under accelerations the accelerometer does not measure purely the gravity vector anymore, thereby distorting the angles calculated from this measurement

- Disturbances of the earth magnetic field caused by (intensive) motor usage and/or the environment will distort the angle vector estimate

This is demonstrated in Figure 2a, where some typical angle estimates from the accelerometer and gyroscope measurements are shown for the pitch angle $\phi$, together with the actual angle and Figure $2 \mathrm{~b}$ shows the accelerometer measurements. This shows that the integrated gyroscope measurement is accurate up to the bias, whereas the accelerometer angle does not show a bias in steady state but has more noise as well as large distortions when accelerations are present. As shown in the figure, for the complementary filter, a small $\alpha$ will filter significantly the non-stationary noise of the vector estimates but will not be robust to the offset of the estimate obtained with the gyroscope. In turn, a large $\alpha$ will be too sensitive to the non-stationary noise of the accelerometer. This motivates making $\alpha$ time-dependent and adapt it with respect to perceived properties of $N_{v}$ (we will use a similarity based approach in the sequel).

\section{Problem Formulation}

The problem considered in this paper is that of estimating the attitude of a body-fixed frame with respect to that of the inertial frame, parametrized by the rotation matrix $R$, subject to the kinematics in (1) and given the vector and gyroscope measurements given by (12) and (13), respectively. In particular, the attitude estimate should be robust to disturbances acting on the vector measurements. 


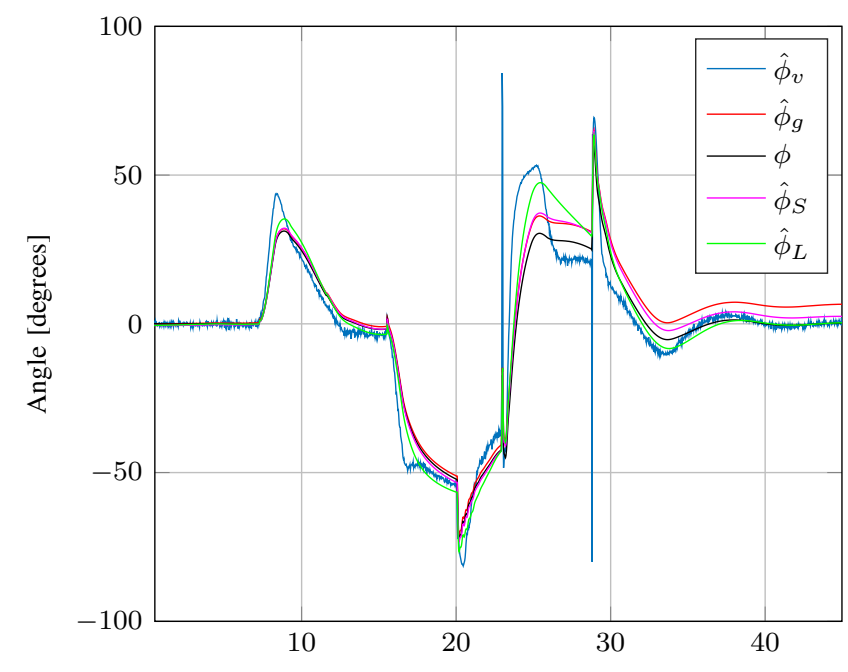

(a)

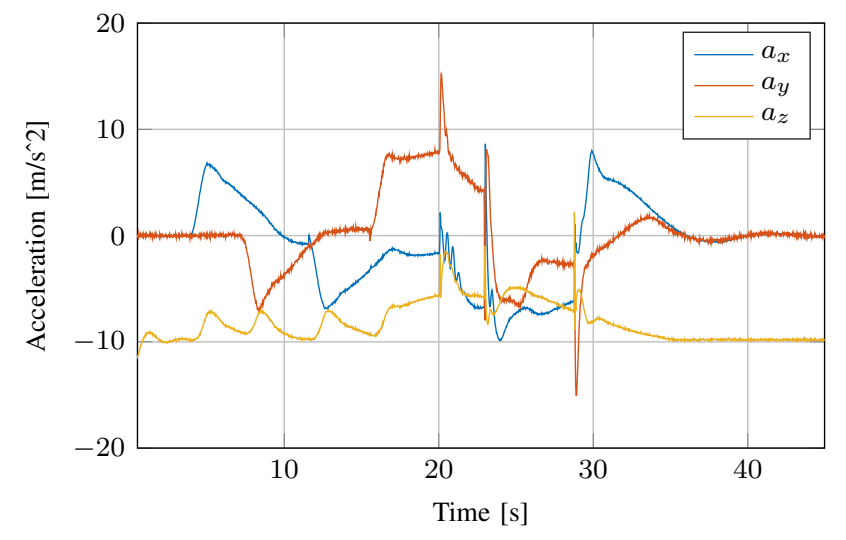

(b)

Fig. 2. Motivation for adaptive gain strategy using data from a simulated quadcopter. In the plots: (a) Typical angle estimates obtained using the vector $\left(\hat{\phi}_{v}\right)$ and gyroscope $\left(\hat{\phi}_{g}\right)$ measurements, together with the actual angle $(\phi)$ and the estimate given by the complementary filter with a small $\alpha\left(\hat{\phi}_{S}\right)$ and a large $\alpha\left(\hat{\phi}_{L}\right)$. (b) Associated accelerometer signals, showing the connection between accelerations and deviations for the vector estimate. Note that for the complementary filter, a small $\alpha$ will filter significantly the non-stationary noise of the vector estimates but will be not robust to the offset of the estimated obtained with the gyroscope and a large $\alpha$ will be too sensitive to the non-stationary noise of the accelerometer.

\section{Proposed Adaptive Method}

In order to address the shortcomings described in the previous section we propose an adaptive gain complementary filter, where the gain is adapted according to the reliability of the angle vector measurement, $\lambda_{v}$.

The adaption is based on the following observation: If the angular estimate achieved from integrating the gyroscope measurement is similar to the angular estimate determined from the accelerometer and magnetometer measurements over a time window, then the accelerometer and magnetometer measurements are not distorted.

In order to quantify the similarity between the angle vector and gyroscope measurement, we introduce a similarity measure

$$
S(t)=\min \left(\bar{S}, \min _{c} J(c, t)\right)
$$

where $0<\bar{S}<\infty$ and

$$
J(c, t)=\sqrt{\int_{t-h}^{t}\left(\lambda_{v}(\tau)-\lambda_{g}(\tau)-c\right)^{2} \mathrm{~d} \tau},
$$

which depends on the signal values $\lambda_{g}$ and $\lambda_{v}$ in a moving time window of length $h$, i.e., in the interval $\tau \in[t-h, t]$. It can be shown that the minimizer of (19) is simply the mean value of the two vectors over the window length, i.e.

$$
c^{*}(t):=\underset{c}{\arg \min } J(c, t)=\frac{1}{h} \int_{t-h}^{t}\left(\lambda_{v}(\tau)-\lambda_{g}(\tau)\right) \mathrm{d} \tau .
$$

This results in very fast calculation times for $S(t)$. The adaptive gain is then defined as

$$
\alpha(t)=\bar{\alpha} e^{-K S(t)},
$$

where $\bar{\alpha}>0$ and $K>0$. This results in

$$
0<\underline{\alpha} \leq \alpha(t) \leq \bar{\alpha} \quad \forall t \in \mathbb{R}_{\geq 0},
$$

where $\underline{\alpha}=\bar{\alpha} e^{-K \bar{S}}$.

In order to show convergence of the observer with adaptive gain in the absence of noise we look at the time domain representation of the linear complementary filter as presented in (17) with the adaptive gain (22), leading to

$$
\dot{\hat{\lambda}}=\omega_{g}+\alpha(t)\left(\lambda_{v}-\hat{\lambda}\right) .
$$

We introduce the Lyapunov function

$$
V(\tilde{\lambda})=\frac{1}{2} \tilde{\lambda}^{2}
$$

where $\tilde{\lambda}=\lambda-\hat{\lambda}$ is the angle error. Taking the derivative of (25) with respect to time gives

$$
\dot{V}=-\alpha(t) \tilde{\lambda}^{2}-n_{\omega} \tilde{\lambda}-\alpha(t) n_{v} \tilde{\lambda}
$$

which is negative definite in the absence of noise, so that we can conclude that the estimation error converges to zero in the absence of noise and is input-to-state (ISS) stable with noise considering the noise properties as discussed in Section II.

\section{Adaptive Complementary Filter on SO(3)}

Now that the rationale behind our approach was shown for the linear case, we move to presenting the ACF directly on $S O(3)$. The problem of estimating the attitude directly on the special orthogonal group can be formulated as determining an estimate $\hat{R} \in S O(3)$ of the rotation matrix $R$ that rotates the body-fixed frame $\mathcal{B}$ to the inertial frame $\mathcal{I}$, from measurements provided by the accelerometer, gyroscope and magnetometer of the IMU. If we define the estimator frame as $\mathcal{E}$, then $\hat{R}$ rotates $\mathcal{E}$ to $\mathcal{I}$.

The attitude kinematics are repeated here for convenience

$$
\dot{R}=R \omega_{\times}
$$

In [11] a passive complementary filter on $S O(3)$ was introduced, which we will expand by introducing a similarity 
measure for the special orthogonal group. The proposed estimator in [11] has the following kinematics

$$
\dot{\hat{R}}=\hat{R}\left(\left(\omega_{g}\right)_{\times}+k_{p} \mathbb{P}_{a}(\tilde{R})\right),
$$

where $k_{p}>0$ is the observer gain, $\mathbb{P}_{a}(H)=\frac{1}{2}\left(H-H^{T}\right)$ is the anti-symmetric matrix projection operator in matrix space and

$$
\tilde{R}=\hat{R}^{T} R_{v} \in S O(3)
$$

is the rotation from the estimator frame to the inertial frame and can be considered the error of the observer in $S O(3)$. The complementary behavior of the estimator in (28) becomes clear by comparing the block-diagram representation in Figure 3 with the classical complementary filter in Figure 1.

As in the linear case, the estimator suffers from disturbances applied to the vector measurements, for which we present a similar adaptation scheme. In order to compare the gyroscope and vector measurements on $S O(3)$ we use the quaternion representations of each measurement. Let $q_{v}$ and $q_{g}$ denote the quaternions representing the rotation calculated from the angle vector and gyroscope measurements, respectively, then we define the similarity measure, with a slight abuse of notation, as

$$
S(t)=\min \left(\bar{S}, \min _{c} J(c, t)\right),
$$

where $0<\bar{S}<\infty$,

$$
J(c)=\sqrt{\sum_{i=1}^{4} \alpha_{i} \int_{t-h}^{t}\left|q_{v, i}(\tau)-q_{g, i}(\tau)-c_{i}\right| \mathrm{d} \tau}
$$

$c=\left(c_{1}, c_{2}, c_{3}, c_{4}\right), \alpha_{i}>0$ are positive constants and $q_{v, i}$ denotes the $i$-th element of the quaternion. The adaptive gain is again defined as

$$
k_{p}(t)=\bar{k} e^{-\xi S(t)},
$$

with $\bar{k}>0$ and $\xi>0$, so that

$$
0<\underline{k} \leq k_{p}(t) \leq \bar{k} \quad \forall t \in \mathbb{R}_{\geq 0}
$$

with $\underline{k}=\bar{k} e^{-\xi \bar{S}}$.

In order to analyze the convergence of the estimator with adaptation we follow the same steps as in [13]. First, note that the goal of the observer is to drive the estimation error to $\tilde{R} \rightarrow I_{3}$, since this means that $\hat{R}$ and $R$ coincide. We adopt the Lyapunov function

$$
E_{t}=\frac{1}{2}\left\|I_{3}-\tilde{R}\right\|_{F}^{2}=\frac{1}{2} \operatorname{tr}\left(I_{3}-\tilde{R}\right)
$$

and its derivative with respect to time is

$$
\dot{E}_{t}=-k_{p}(t)|\operatorname{vex}(\mathbb{P}(\tilde{R}))|^{2},
$$

which is negative definite so that we can conclude, using similar arguments as in [13], that the estimation error converges to zero. This shows that the filter in [13] can be extended to an adaptive filter, and in particular to the one we propose, without affecting the convergence guarantees.

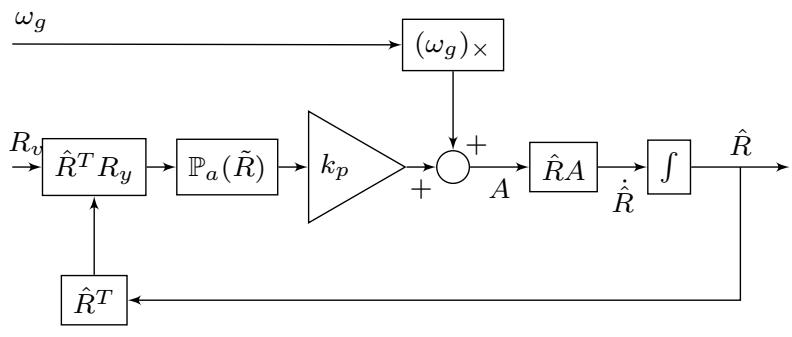

Fig. 3. Passive complementary filter on $S O(3)$

\section{Simulations}

In order to demonstrate the effectiveness of our approach simulations were performed using a model of a quadcopter and compared to the non-adaptive passive complementary filter as proposed in [13].

Figure 4 shows the first simulation, where for the adaptive filter the maximum gain was set to $\bar{k}=0.3\left[\mathrm{rad} \cdot \mathrm{s}^{-1}\right]$, the gain factor was set to $\xi=0.4[-]$ and the window length was set to $h=0.5[s]$ and for the non-adaptive filter the gain was fixed to $k_{p}=0.1\left[\mathrm{rad} \cdot \mathrm{s}^{-1}\right]$. The second simulation is shown in Figure 5, where the setting were $\bar{k}=1\left[\mathrm{rad} \cdot \mathrm{s}^{-1}\right]$, $\xi=2[-], h=0.5[s]$ and $k_{p}=1\left[\mathrm{rad} \cdot \mathrm{s}^{-1}\right]$. The trajectories in both figures are the same.

In both figures the top and middle plot show the angular estimates resulting from the non-adaptive (blue) filter, adaptive filter (green), gyroscope integration (red) and the actual angle (dashed black) for the pitch and roll angles, respectively. The bottom plot shows the adaptive gain (green) together with the fixed gain (blue) over time.

Together the figures display the advantage of the adaptive over the non-adaptive filter. In Figure 4 the fixed gain $k_{p}$ is set low, thus giving more priority towards the gyroscope measurements, resulting in good estimates during accelerations but a poor convergence in steady-state, resulting in a large steady-state deviation. In contrast, the larger gain setting of Figure 5 results in a better performance in steady-state conditions, but suffers a great deal from deviations during accelerations.

The proposed adaptive filter performs better than its nonadaptive counterpart in both simulations. It allows for a larger initial gain, resulting in better steady-state behavior, whilst still allowing the gain to be lowered using the adaptation rule from (32) in order to avoid deviations during accelerations. This essentially gives the user more knobs to turn in order to adjust the filter to the behavior of the sensors of the IMU.

\section{COnClusions \& Future Work}

The problem of attitude estimation considering disturbances acting on the vector measurements was solved using an adaptation scheme for the passive complementary filter directly on the special orthogonal group. The adaptation scheme presented is this paper was proven to converge and simulations results showed that the adaptation scheme performs as expected, showing the advantages compared to the non-adaptive filter. 
CONFIDENTIAL. Limited circulation. For review only.

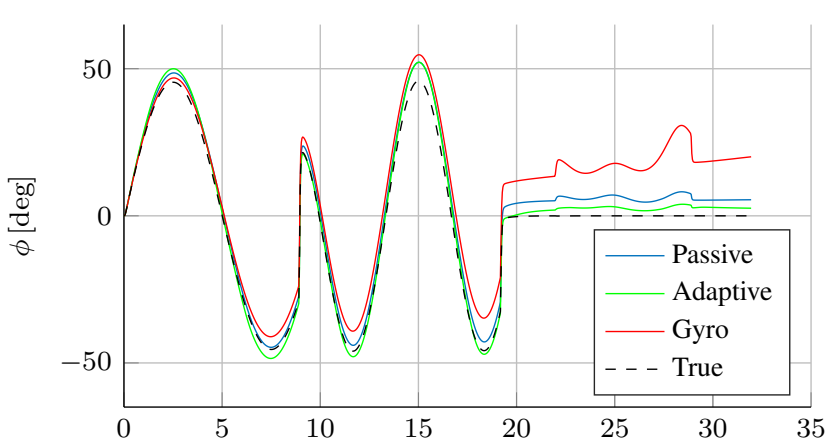

(a)

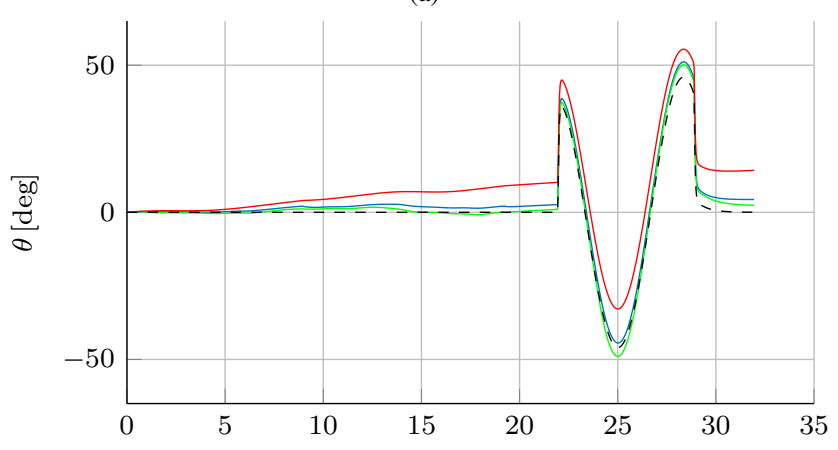

(b)

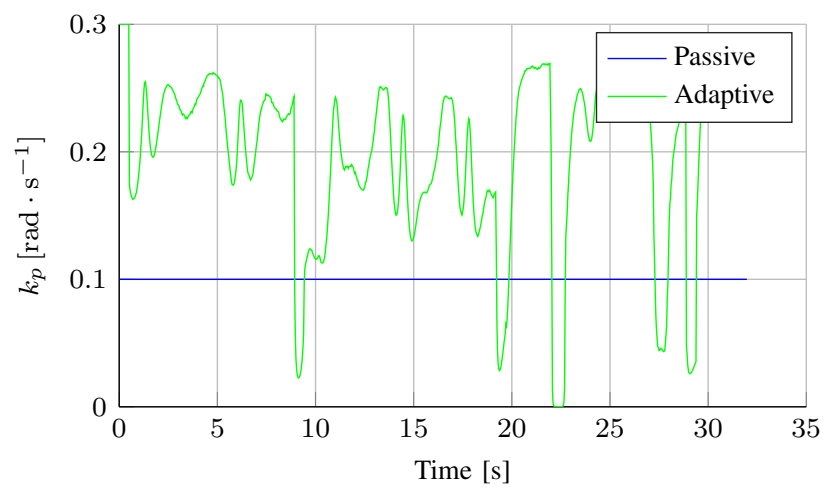

(c)

Fig. 4. Simulation results for $k_{0}=0.3\left[\mathrm{rad} \cdot \mathrm{s}^{-1}\right], \xi=0.4[-], h=$ $0.5[s], k_{p}=0.1\left[\mathrm{rad} \cdot \mathrm{s}^{-1}\right]$. The top and middle plot show the angular estimates resulting from the non-adaptive (blue) and adaptive filter (green), gyroscope integration (red) and the actual angle (dashed black) for the pitch and roll angles, respectively. The bottom plot shows the adaptive (green) and fixed gain (blue) over time.

Future work will include experimental testing of our method, as well as the use of the adaptation scheme on the passive complementary filter with bias correction and other, more advanced complementary filters that have been proposed in the literature. Moreover, we plan to use different adaptation schemes based on machine learning.

\section{REFERENCES}

[1] S. O. H. Madgwick, A. J. L. Harrison, and R. Vaidyanathan, "Estimation of IMU and MARG orientation using a gradient descent algorithm," in 2011 IEEE International Conference on Rehabilitation Robotics, June 2011, pp. 1-7.

[2] H. J. Luinge, P. H. Veltink, and C. T. M. Baten, "Estimation of orientation with gyroscopes and accelerometers," in Proceedings of the First Joint BMES/EMBS Conference. 1999 IEEE Engineering in

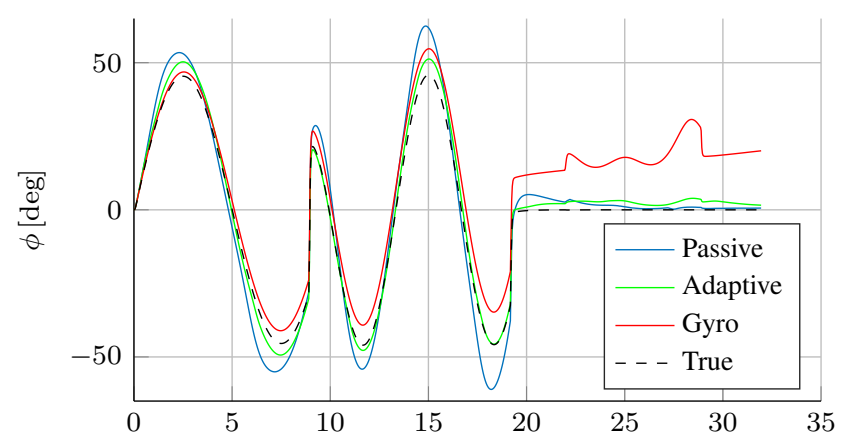

(a)

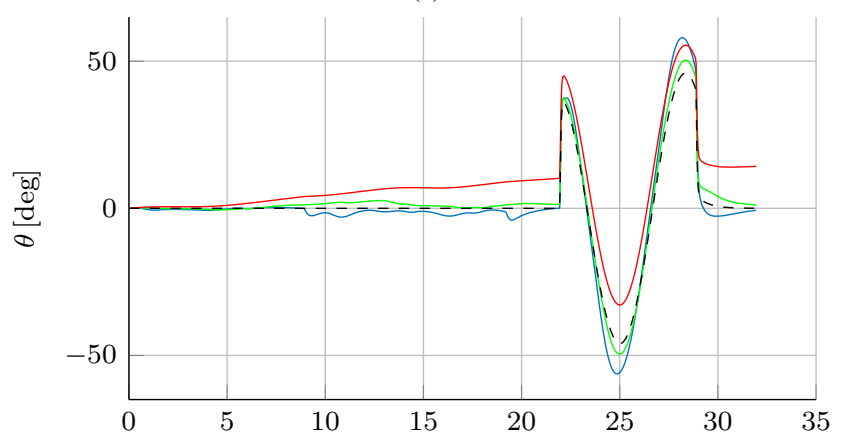

(b)

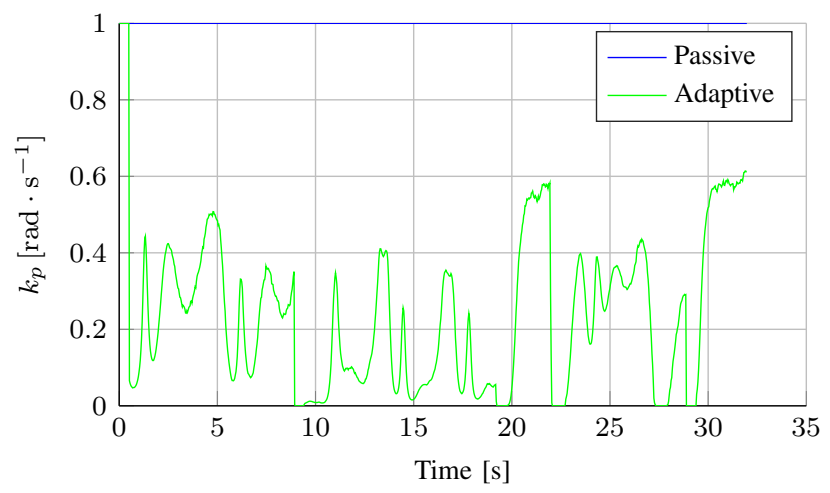

(c)

Fig. 5. Simulation results for $k_{0}=1\left[\mathrm{rad} \cdot \mathrm{s}^{-1}\right], \xi=2[-], h=0.5[s]$, $k_{p}=1\left[\mathrm{rad} \cdot \mathrm{s}^{-1}\right]$. The top and middle plot show the angular estimates resulting from the non-adaptive (blue) and adaptive filter (green), gyroscope integration (red) and the actual angle (dashed black) for the pitch and roll angles, respectively. The bottom plot shows the adaptive (green) and fixed gain (blue) over time. 
Role of Robotics in the the Next Millennium (Cat. No.01CH37180), vol. 4, 2001, pp. 2003-2011 vol.4.

[7] S. Sabatelli, M. Galgani, L. Fanucci, and A. Rocchi, "A double-stage kalman filter for orientation tracking with an integrated processor in 9-d imu," IEEE Transactions on Instrumentation and Measurement, vol. 62, no. 3, pp. 590-598, March 2013.

[8] S. M. Oh, "Multisensor fusion for autonomous uav navigation based on the unscented kalman filter with sequential measurement updates," in 2010 IEEE Conference on Multisensor Fusion and Integration, Sept 2010, pp. 217-222.

[9] H. G. de Marina, F. J. Pereda, J. M. Giron-Sierra, and F. Espinosa, "Uav attitude estimation using unscented kalman filter and triad," IEEE Transactions on Industrial Electronics, vol. 59, no. 11, pp. 4465-4474, Nov 2012.

[10] A. J. Baerveldt and R. Klang, "A low-cost and low-weight attitude estimation system for an autonomous helicopter," in Proceedings of IEEE International Conference on Intelligent Engineering Systems, Sep 1997, pp. 391-395.

[11] R. Mahony, T. Hamel, and J. M. Pflimlin, "Complementary filter design on the special orthogonal group so(3)," in Proceedings of the 44th IEEE Conference on Decision and Control, Dec 2005, pp. 14771484.

[12] T. Hamel and R. Mahony, "Attitude estimation on so[3] based on direct inertial measurements," in Proceedings 2006 IEEE International Conference on Robotics and Automation, 2006. ICRA 2006., May 2006, pp. 2170-2175.

[13] R. Mahony, T. Hamel, and J. M. Pflimlin, "Nonlinear complementary filters on the special orthogonal group," IEEE Transactions on Automatic Control, vol. 53, no. 5, pp. 1203-1218, June 2008.

[14] H. Simon, "Adaptive filter theory," Prentice Hall, vol. 2, pp. 478-481, 2002.

[15] W. Li and J. Wang, "Effective adaptive kalman filter for mems$\mathrm{imu} /$ magnetometers integrated attitude and heading reference systems," Journal of Navigation, vol. 66, no. 1, p. 99113, 2013.

[16] K. D. Sebesta and N. Boizot, "A real-time adaptive high-gain ekf, applied to a quadcopter inertial navigation system," IEEE Transactions on Industrial Electronics, vol. 61, no. 1, pp. 495-503, Jan 2014.

[17] A. L. da Silva and J. J. da Cruz, "Fuzzy adaptive extended kalman filter for uav ins/gps data fusion," Journal of the Brazilian Society of Mechanical Sciences and Engineering, vol. 38, no. 6, pp. 1671-1688, Aug 2016. [Online]. Available: https://doi.org/10.1007/s40430-0160509-7

[18] A. A. Neto, D. G. Macharet, V. C. da Silva Campos, and M. F. Montenegro Campos, "Adaptive complementary filtering algorithm for mobile robot localization," Journal of the Brazilian Computer Society, vol. 15, no. 3, pp. 19-31, Sep 2009. [Online]. Available: https://doi.org/10.1007/BF03194503

[19] T. S. Yoo, S. K. Hong, H. M. Yoon, and S. Park, "Gain-scheduled complementary filter design for a mems based attitude and heading reference system," Sensors, vol. 11, no. 4, pp. 3816-3830, 2011. [Online]. Available: http://www.mdpi.com/1424-8220/11/4/3816

[20] R. G. Valenti, I. Dryanovski, and J. Xiao, "Keeping a good attitude: A quaternion-based orientation filter for imus and margs," Sensors, vol. 15, no. 8, pp. 19302-19330, 2015. [Online]. Available: http://www.mdpi.com/1424-8220/15/8/19302

[21] P. Marantos, Y. Koveos, and K. J. Kyriakopoulos, "Uav state estimation using adaptive complementary filters," IEEE Transactions on Control Systems Technology, vol. 24, no. 4, pp. 1214-1226, July 2016.

[22] G. Wahba, "A least squares estimate of satellite attitude," SIAM Review, vol. 7, no. 3, pp. 409-409, 1965. [Online]. Available: https://doi.org/10.1137/1007077

[23] R. Kottath, P. Narkhede, V. Kumar, V. Karar, and S. Poddar, "Multiple model adaptive complementary filter for attitude estimation," Aerospace Science and Technology, vol. 69, no. Supplement C, pp. 574 - 581, 2017. [Online]. Available: http://www.sciencedirect.com/science/article/pii/S1270963816303418

[24] S. Garrido-Jurado, R. M. noz Salinas, F. Madrid-Cuevas, and M. Marín-Jiménez, "Automatic generation and detection of highly reliable fiducial markers under occlusion," Pattern Recognition, vol. 47, no. 6, pp. 2280 - 2292, 2014. [Online]. Available: http://www.sciencedirect.com/science/article/pii/S0031320314000235 